\title{
Die zukünftige Geschichte der Lepra
}

\author{
The Forthcoming History of Leprosy
}

Autoren

Institute
M. Hundeiker ${ }^{1}$, I. D. Bassukas ${ }^{2}$

${ }^{1}$ Lepramuseum Münster

${ }^{2}$ Dermatologische Universitätsklinik loannina
Bibliografie

DOI http://dx.doi.org/

10.1055/s-0034-1365743

Online-Publikation: 5.6.2014

Pneumologie 2014; 68: 613-618

(c) Georg Thieme Verlag KG

Stuttgart · New York

ISSN 0934-8387

Korrespondenzadresse Prof. Dr. Max Hundeiker Immelmannstraße 16 48157 Münster

max@hundeiker.de

\section{Einleitung $\nabla$}

Jahrzehntelang vor allem im Umfeld der WHO wiederholte Ankündigungen einer baldigen Ausrottung der Lepra waren nie wissenschaftlich vertretbar. Für die „Eradikation“ fehlen die Voraussetzungen. Die Bezeichnung „Elimination“ für bloße Reduktion der Prävalenz unter 1:10000 ist irreführend. Erreichbar ist nur Kontrolle der Lepra. Entdeckung und Behandlung aller Infizierten ist kaum möglich. Mehr Hoffnung knüpft sich an die Entwicklung von Impfungen. Aber möglicherweise werden sich von selbst genetische Anpassungen durchsetzen, die z.B. in Mitteleuropa schon im Beginn der Neuzeit die Lepra erlöschen ließen.

\section{Heutiger Kenntnisstand \\ $\nabla$}

Ähnlich wie Tuberkelbakterien gelangen auch die Erreger der Lepra im Wesentlichen durch Tröpfcheninfektion der Atemwege in den Organismus $[1,2]$. Die Lepra manifestiert sich aber in mehr peripheren Regionen. Die verbreitete Vorstellung, sie sei längst überwunden, trifft nicht zu [3]. Ihr scheinbar völliges Verschwinden aus Mitteleuropa im Beginn der Neuzeit trotz Fehlens einer wirksamen Behandlung war bis in die jüngste Zeit rätselhaft [4]. In Randzonen Europas war sie präsent geblieben. Im Westen Norwegens war ihre Prävalenz im 19. Jahrhundert hoch und schien noch zuzunehmen. Wenig später ging sie auch dort zurück. Dazu dürften damalige Mittel und die unvollkommene Isolierung in Leprosorien kaum beigetragen haben [4]. Inzwischen hatte in Bergen 1873 Gerhard Henrik ArmauerHansen (den Familiennamen der Großmutter mütterlicherseits hatte er seinem hinzugefügt, um sich von anderen Hansens zu unterscheiden) die Ursache der Seuche entdeckt, das Mycobacterium leprae.
Heute, über 140 Jahre nach Entdeckung des Bakteriums und 40 Jahre nach der durch das Deutsche Aussätzigen-Hilfswerk geförderten Einführung der ersten "Multidrug-Therapy“ (MDT) durch Freerksen [5, 6], ähnelt die Verteilung neuer Fälle in aktuellen Endemiegebieten der Situation in Europa zur Zeit Hansens: Zwischen Regionen mit zurückgehenden Inzidenzdaten persistieren Enklaven mit hoher Durchseuchung und Prävalenz $[7,8]$. Andererseits läuft weltweit die Entwicklung, die einst in Mitteleuropa der Lepra ihren Schrecken genommen hat, weiter $[9,10]$. Sie ist, wie viele Veröffentlichungen erkennen lassen, außerhalb der engeren Fachwelt wenig bekannt. Deshalb erscheint es lohnend, ihre wichtigsten Aspekte hier noch einmal ausschnittweise zusammenzufassen.

Mycobacterium leprae, die von Hansen entdeckte Erregerspezies, weist mehrere immungenetisch und molekularbiologisch distinkte Typen auf. Eine zweite Lepra erregende Spezies wurde vor wenigen Jahren als Mycobacterium lepromatosis abgegrenzt [11-13]. Die Mykobakterien umfassen mehr als 120 bekannte Spezies. Viele davon leben im Boden. Als Krankheitserreger sind Tuberkelbakterien am wichtigsten. Leprabakterien haben sich stärker als die bekannten Typen des Mycobacterium tuberculosis an das Leben im Menschen angepasst. Bei ihnen sind für diese Existenzform nicht gebrauchte Teile ihres genetischen Materials, das alle Lebensvorgänge steuert, verloren gegangen [14]. Beispielsweise hat das Genom der Tuberkel-Bakterien eine Länge von 4411522 Basen-Paaren mit 3924 proteincodierenden Sequenzen, das des Mycobacterium leprae nur eine von 3268203 Paaren mit 1604 proteincodierenden Sequenzen. Im Genom der Tuberkelbakterien sind über $90 \%$ Gene, $9 \%$ nicht codierend, bei Leprabakterien aber $50 \%$ Gene, $24 \%$ nicht codierend, $26 \%$ „Pseudogene“, deren biologische Aktivitäten noch kaum bekannt sind. Mycobacterium leprae hat, verglichen mit vielen 
anderen pathogenen Keimen, eine geringe genetische Variabilität [15-17].

Die Anpassungsfähigkeit dieser Bakterien an Umgebung und Wirtsorganismen ist beschränkt. Um dauerhaft zu existieren, sind sie in der „alten Welt“ weitgehend auf Menschen angewiesen. Übertragung auf Mäuse, Igel, Streifenhörnchen gelingt unter speziellen Bedingungen. Züchtung auf Nährböden gelingt gar nicht. Aber bei Affen sind nicht nur Empfänglichkeit, sondern auch natürlich erworbene Infektionen nachgewiesen worden [18], und in der „neuen Welt“ sind Gürteltiere sehr empfänglich [19]. Das liegt sowohl an ihrer genetischen Ausstattung als auch an ihrer für das Mycobacterium leprae günstigen niedrigen Körpertemperatur. Irgendwann müssen einst in Süd- oder Mittelamerika Armadillos (Neunbinden-Gürteltiere) von Menschen mit der aus Europa eingeschleppten Seuche infiziert worden sein. Heute weisen Dasypus novemcinctus und in Brasilien Euphractus sexcinctus eine hohe regional unterschiedliche Durchseuchung auf [20,21]. Gürteltiere sind keine Haustiere, aber sie werden gefangen und gegessen, und sie kommen bei der Jagd nach Kleintieren in Hausgärten. Durch Ausscheidungen und Nasensekret infizierter Tiere gelangen Erreger in den Gartenstaub und werden von Menschen eingeatmet. In den USASüdstaaten sind sie eine wichtige Infektionsquelle [22,23]. Insofern ist der Mensch also nicht einziger Wirt der Leprabakterien, womit schon eine wichtige Voraussetzung für ihre Eradikation fehlt $[24,25]$.

Andererseits haben sich auch Menschen an die Bakterien angepasst: Im Laufe der Geschichte nehmen durch Erbauslese in verschiedenen Gebieten Gruppen zu, deren angeborene Immunabwehr sich erfolgreicher mit dem Mycobakterium leprae auseinandersetzen kann $[9,26,27]$. Um die Abläufe zu verstehen, ist ein kurzer Seitenblick in die Immungenetik nötig [28,29]: Viele Varianten von Erbanlagen für verschiedene an immunologischen Abwehrvorgängen beteiligte Molekülstrukturen gehen einher mit unterschiedlicher Empfänglichkeit für Mykobakterien und unterschiedlicher Ausprägung von Krankheits- und Reaktionstypen: z.B. MHC (Major histocompatibility complex) bzw. HLA-System, Komplement-System, Kollagen Typ III, $\beta$-Defensin, Ficolin, Interferon $\gamma$, Interleukin 4, Nucleotid-oligomerisierungsdomain 2, Parkin 2, Mannose-bindendes Lektin usw. sowie „Tollähnliche Rezeptoren“. Zellen der Immunabwehr erkennen bakterielle Strukturen als fremd mit Pathogen Recognition Receptors, (PRR), ihre „Außenhaut“ durchsetzenden Molekülen. Dazu gehören die Toll Like Receptors (TLR, signaltransduktions-vermittelnde PRR). Sie haben gemeinsame Strukturmerkmale: N-terminale leucinreiche LRR-Sequenzen (Leucin-Rich-Repeats) und „TIRDomänen“. Sie können aufgrund schlüsselähnlich dazu passender Formen mit bestimmten „fremden“ Strukturen reagieren und daraufhin in der Zelle weitere Reaktionen in Gang setzen. Sie können mit jeweils unterschiedlichen PAMPs (Pathogen Associated Molecular Patterns) reagieren. Das sind molekulare Strukturen, die an der Oberfläche von Krankheitserregern vorkommen. TLR in der Plasmamembran der Zelloberfläche (TLR 2,4-6) reagieren zum Beispiel mit Lipopolysacchariden, bakteriellen Lipoproteinen, viralen Proteinen. TLR 3,7-9 erkennen bakterielle und virale Nukleinsäuren. Die durch TLR vermittelte Immunerkennung ist ein evolutionär sehr alter, genetisch „hoch konservierter" Mechanismus. Trotzdem ist die Ausstattung mit solchen Erkennungsmolekülen nicht bei allen Menschen gleich. Die regionale Verteilung genetischer Polymorphismen (Variationen im molekularen Strukturaufbau mit variablen funktionellen Konsequenzen) der Toll-Like-Rezeptor-Gene, welche Proteine codieren, die an der Bakterienerkennung durch Zellen der Immunabwehr mitwirken, lässt den Schluss zu, dass Lepra ein SelektionsdruckFaktor in der jüngeren Evolution der Menschheit war [9].

Ansteckung mit Lepra geschieht meist durch Tröpfcheninfektion mit Atemluft. Schon nach kurzem Gesprächskontakt mit Kranken ist mit der PCR (Polymerase Chain Reaction) häufig Erreger-DNA in der Nasenschleimhaut nachzuweisen. Gelegentlich können die Bakterien auch durch Sekret aus Geschwüren und Hautläsionen, Absonderungen aller Art, sogar Muttermilch, mit Sekret verschmutzte Wäsche oder Geschirr, auch durch Staub und Erde übertragen werden. Die verbreitete Vorstellung, Lepra sei wenig infektiös, ist ebenso falsch wie die, dass erst nach langen Kontakten Infektionen zustande kämen und dass nur lepromatöse bzw. multibakterielle Formen ansteckend seien. Sie ist fortgeerbt aus Zeiten, in denen noch keine molekularbiologische Diagnostik verfügbar war und an inapparente Infektionen wenig gedacht wurde. Noch ungeklärt ist, inwieweit die Dauer der Latenz von der aufgenommenen „Bakterienlast“ abhängt. Enge Kontakte von Nase zu Nase, wie z.B. in einer mit Lepra endenden erotischen Kurzgeschichte von Roald Dahl beschrieben [Switch Bitch (1974; Kuschelmuschel, ISBN 3-499-23255-3)], vergrößern aber vermutlich die Gefahr der Aufnahme großer Bakterienmengen. Die Nasenschleimhaut ist wichtigste Eintrittspforte und auch wichtigste Quelle der Erregerausbreitung auf andere. Doch auch die weitere Umgebung unbehandelter Infizierter enthält reichlich Mycobakterien [30-32].

Durchaus nicht jeder, der mit dem Erreger infiziert wird, wird dadurch krank [9,28,33-35]. Ob jemand erkrankt, liegt weniger, als das in der Laienliteratur oft dargestellt wird, an Armut, viel mehr an Hygiene, Sauberkeit, Intensität der Kontakte mit schon Infizierten. Möglicherweise haben Koinfektionen mit Tuberkelbakterien einen schützenden Einfluss. Einige Autoren vermuten als Ursache dafür eine gegen Mycobacterium leprae wirksame „Kreuzimmunität“. Andererseits wird auch eine aus der Koinfektion resultierende erhöhte Sterberate diskutiert [36,37]. Aber entscheidend ist das alles nicht, sondern die genetisch bedingte Empfänglichkeit für die Krankheit [28,33-38]. In Mitteleuropa ist zu Beginn der Neuzeit durch Erbauslese der Anteil gegen Lepra-Erreger widerstandsfähiger Individuen an der Bevölkerung gewachsen [9]. Ähnliche biologische Entwicklungen sind natürlich auch in der Zukunft und in anderen Weltgegenden, wo Prävalenz und vor allem Inzidenz der Seuche noch höher sind, möglich. Aus solchen Ländern stammt ein großer Teil der jetzt in Europa zu behandelnden Kranken.

Weit über 90\% der heutigen Mitteleuropäer sind gegen Lepraerreger „resistent“ und erkranken nach Infektion nicht. Menschen europäischer Abstammung sind größtenteils homozygot in einer bestimmten TLR (Toll Like Receptor)-1 Variante, die vor Erkranken durch Leprabakterien schützt $[9,27-29,34,35,38]$. Das bedeutet jedoch nicht, dass sie nicht Erreger weitergeben können. Hierzu ein Einzelbeispiel: Wir konnten beim Kongress der Deutschen Dermatologischen Gesellschaft 1965 eine Kranke vorstellen, die nie aus ihrer von Lepra freien Heimat herausgekommen war. Aber sie war kurze Zeit Dienstmädchen in der Familie eines Jahre zuvor in Indochina eingesetzten französischen Offiziers. Bei einem früheren Hausangestellten in Indochina ist viele Jahre später Lepra diagnostiziert worden. Niemand aus der französischen Überträgerfamilie ist während jahrzehntelanger Kontrollen je erkrankt. Anscheinend geht die Auseinandersetzung zwischen Erregern und Organismus bei gegen Lepra resistenten Menschen in dauernde Symbiose (Persistenz oder sogar Gedeihen von Bakterien in Menschen ohne Erkrankung) über. 
Wenn später dies Verhältnis durch konsumierende Leiden (z. B. Leukose, AIDS) oder medikamentöse Immunsuppression gestört wird, kann die Krankheit unerwartet ausbrechen [39].

Ein für Lepra empfänglicher Mensch erkrankt nach Infektion mit Mycobacterium leprae erst nach einer Inkubationszeit von zumindest Monaten, oft aber Jahrzehnten, denn die Erreger wachsen extrem langsam. Sie teilen sich höchstens einmal in 14 Tagen und gedeihen am besten bei relativ niedrigen Temperaturen um $33^{\circ} \mathrm{C}$. Deshalb sind oft kühle Körperbereiche, wie Naseninneres, Nase, Stirn und Ohren, Hände und Füße, betroffen. Die Bakterien befallen besonders Schwannsche Zellen (Hüllzellen um Nervenfasern), Endothelien (Auskleidung der Adern) und Makrophagen („Fresszellen“). Fresszellen schaffen es beim Leprakranken teilweise nicht, die Bakterien zu vernichten. Diese werden zwar aufgenommen („Phagozytose“) und in „Endosomen“ eingeschlossen. Die werden aber nicht mit Lysosomen verschmolzen, welche ihren Inhalt verdauen. Mycobakterien können das enzymatisch hemmen. Sie können sich dann sogar in Endosomen vermehren. In der „Verdauung“ gestörte Fresszellen teilen sich anfangs noch, wuchern, schwellen an zu Epitheloidzellen. Durch gestörte Teilung entstehen mehrkernige Riesenzellen, darunter „VirchowZellen" mit schaumigem Zellkörper. Die Bläschen darin sind voll Bakterien, die sich bei Zerfall der Zelle entleeren. Epitheloidzellen und Riesenzellen bilden Granulome, die makroskopisch (mit bloßem Auge) als verhärtete Knötchen und Knoten erkennbar werden. Schon von außen kann man oft solche Nervenverdickung fühlen, z.B. am Ulnar-Nerven am Ellenbogen. Befallene Schwannsche Zellen der Nerven gehen teils zugrunde, teils wird ihr Genom verändert, sie wachsen und dienen Erregern als Substrat. Erst wenn diese veränderten Zellen entzündlich zerstört und abgebaut werden, verlieren die Nervenfasern ihre „Isolierung" ganz und funktionieren nicht mehr, auch wenn sie selbst bis dahin unzerstört waren - manchmal auch noch lange nach erfolgreicher Behandlung der Infektion [40-43]. Einzelne Autoren grenzen deshalb von der eigentlichen Infektionskrankheit Lepra diese Lepra-Neuritis ab als eine immunologische Folge-Krankheit $[44,45]$.

Einzelheiten über Verlauf, diagnostische Möglichkeiten und Komplikationen müssen hier nicht ausgebreitet werden, doch einige grundsätzliche Aspekte sind für das allgemeine Verständnis wichtig: Krankheitsbild und Verlauf der Lepra hängen ab von der „Abwehrlage“. Nosologisch unterscheidet man „paucibazilläre“ bakterienarme Formen bei relativ effektiver und „multibazilläre“ bakterienreiche bei schwacher Immunabwehr. Klinisch entsprechen als Hauptformen der paucibazillären die tuberkuloide, der multibazillären die lepromatöse Lepra. „Borderline“ Lepra entwickelt Merkmale beider Formen. Die Lepra indeterminata bei besonders guter Abwehr hat nur geringe, oft von selbst heilende Veränderungen. Die Symptomatik ist anfangs geprägt durch Ausfall sensibler Hautnerven, durch rötliche und vor allem weißliche Flecken der Haut mit Verlust der Pigmentierung. Später kommen dickere rötlich-bräunliche Hautinfiltrate hinzu. Die Flecken werden für Temperatur und schließlich Schmerzreiz, wie z. B. Nadelstiche, unempfindlich. Das ist oft ein wichtiger erster Hinweis für das Bemerken der Krankheit. Im Prinzip können zwar überall entzündliche Infiltrate entstehen, aber entsprechend der Vorliebe dieser Erreger für kühle Temperaturen sind schwerpunktmäßig gut belüftete, kühle, und frei getragene Gegenden betroffen: Hände und Füße, Gesicht, obere Atemwege, Hoden. An den Händen und Füßen führt Lepra wegen Befalls der zugehörigen Nerven, Verödung der Adern und Rückganges nicht mehr von intakten Nerven versorgter Muskeln zu typischer

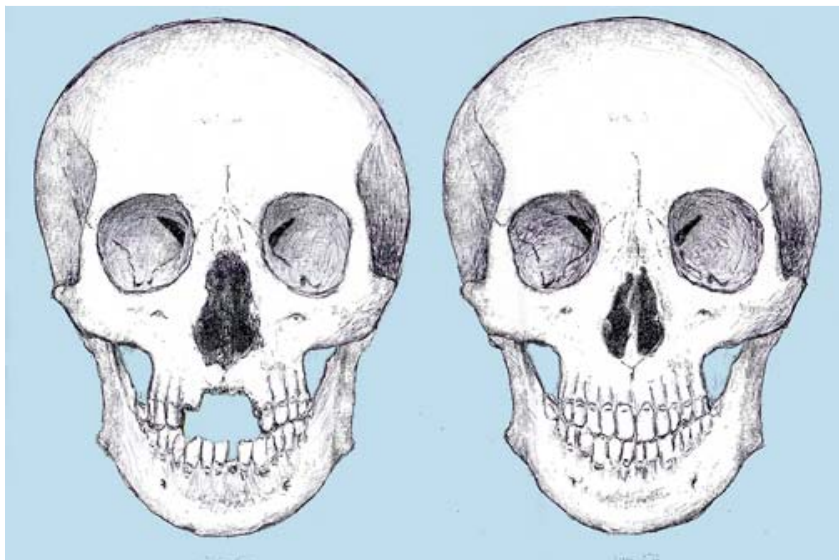

Abb. 1 Schematische Darstellung charakteristischer Lepramerkmale an alten Schädeln (links) mit Zerstörungen im Umfeld der oberen Atemwege im Vergleich zum Normalbefund (rechts). Eine eingehendere Darstellung der osteoarchäologischen Befunde geben z. B. Boldsen JL, Rasmussen KL, Riis T, Dittmar M, Weise S: Schleswig: medieval leprosy on the boundary between Germany and Denmark. Anthropol Anz. 2013; 70: 273-287.

„Klauenstellung“ der Finger und Zehen. Die Greiffähigkeit geht dadurch verloren. An empfindungslosen Fingern und Zehen entstehen nach geringsten, unbemerkten Verletzungen an Händen und Füßen und Infektion mit anderen Keimen nicht heilende Geschwüre. Finger oder Zehen faulen sozusagen bei lebendigem Leibe ab durch fortschreitende entzündliche Zerstörung. Frühe Veränderungen werden nicht selten anfangs als „rheumatisch“ verkannt. Im Gesicht treten Infiltrate oft über Jochbein und Augenbrauen auf. Augenbrauen fallen aus (Madarosis), Kranke bekommen ein „Löwengesicht“ (Facies leonina) mit Verlust der Mimik (Facies antonina), Vernarbung der Augen, Erblindung.

Oberflächengebiete mit niedriger Temperatur sind außen die Ohren, die Jochbeingegend, Lippen und Nase, innen die Nasenscheidewand, Muscheln, Umgebung des Naseninneren, Larynx [46]. Auch die Temperatur am harten Gaumen liegt mit etwas über $27^{\circ} \mathrm{C}$ viel niedriger als die durchschnittliche Hautoberflächentemperatur von über $33^{\circ} \mathrm{C}$. Dementsprechend entwickeln sich bakterienhaltige Krankheitsherde oft in der Nase (schon bei der mittelalterlichen Lepraschau wurde auf Geschwüre in der Nase geachtet, und hier wird auch heute noch meist zuerst mit Abstrichen nach Erregern gesucht!) sowie an Gaumen, Lippen, Oberkiefer. Die angrenzenden Knochen werden zerstört. Zum Teil sind schon am Lebenden diese Folgen von außen sichtbar: Die Nase sinkt ein. Infolge Zerstörungen in Nasenbein, Siebbein, Oberkiefer und Verlust der Zahn-Alveolen fallen obere Frontzähne aus $[47,48]$. Selbst nach dem Tode geben solche Lepra-bedingten Knochenveränderungen in archäologischen Funden oft erste Hinweise auf Lepra ( $\bullet$ Abb. 1). Oft gelingt auch der Nachweis von Resten des Erbmaterials und anderer Bestandteile, wie spezifischer Lipide von Mykobakterien und ihre Zuordnung zu bestimmten Erregerstämmen. Das kann Schlüsse auf einstige Ausbreitungswege der Seuche ermöglichen.

Wirksame Mittel gegen Lepra sind erst seit der Mitte des 20. Jahrhunderts verfügbar: In den vierziger Jahren wurden Sulfone wie DADPS (Dapsone) eingeführt, 1962 Clofazimin (Lampren), 1963 als erstes gegen Mykobakterien wirksames Antibiotikum Rifampicin. Seit mehr als einem halben Jahrhundert verfügt damit die Medizin über die wichtigsten der heute noch angewendeten Medikamente gegen das Mycobacterium leprae. Der entscheidende Durchbruch ist vor vier Jahrzehnten in Malta gelungen. Mit flä- 
chendeckenden Untersuchungen und der ersten richtigen von Freerksen entwickelten MDT (Multi Drug-Therapy) mit täglicher Gabe von Dapson 0,1, Protionamid 0,35, Isoniacid 0,35, Rifampicin 0,6 in fixer Kombination gelang es, landesweit die Prävalenz der Lepra praktisch auf null zu bringen. Eine wirkliche „Eradikation“" war das natürlich nicht $[5,6,49]$.

Die Behandlung in der Gegenwart ist seit 1981 vereinheitlicht durch Empfehlungen der WHO für die standardisierte MDT mit Diaminodiphenylsulfon (Dapson) und dem Antibiotikum Rifampicin oder bei multibazillärer Lepra Dapson, Clofazimin und Rifampicin. Aber aus ökonomischen Gründen wurden Schemata eingeführt, die pharmakologisch durchaus verbesserungsfähig sind. Unverträglichkeiten wie auch Resistenzen der Erreger gegen einzelne Mittel der MDT sind zunehmend häufig. Aber bei guter medizinischer Kontrolle sind sie durch andere und neue gegen Mykobakterien wirksame Medikamente zu überwinden. Auch mit den vereinfachten MDT-Schemata sind große Erfolge erzielt worden: Der Anteil Kranker an der Bevölkerung, die Prävalenz, nahm rasch ab und damit vor allem auch die Häufigkeit neu aufgetretener Behinderungen durch Krankheitsfolgen [50].

Bald wurde daraufhin immer wieder in Verlautbarungen und Publikationen der WHO und anderer Organisationen mit immer weiter hinausgeschobenen Zeitvorgaben die „Elimination“ oder gar die „Eradikation“ der Seuche angekündigt. Das war voreilig [49-52]. Doch Elimination der Manifestation ist nicht gleich Elimination der Infektion. So ist dem Absinken der Prävalenz keineswegs immer eines der Inzidenz gefolgt: Neue Fälle wurden nicht wirklich weniger [52]: „Transmission is not sufficiently reduced as witnessed by unwavering new case rates".

Wo lagen die Fehler? Zunächst einmal waren wesentliche Voraussetzungen einer Eradikation [24,25] von vornherein nicht gegeben [54], vor allem, weil der Mensch nicht alleiniger Wirt der Erreger ist und die Infektion nicht jeden Infizierten krank macht. Außerdem wurde seit Jahren darauf hingewiesen, dass schon die Art der Datenerfassung zu Fehlinterpretationen führen kann [53,54]: Wo Patienten unter Behandlung gezählt wurden, verringerte sich durch Einführung der Kurzzeit-MDT je nach Erfassungszeitraum und Behandlungszeit scheinbar die Prävalenz. Schon die willkürliche Definition der WHO: „Elimination=Absenkung der Prävalenz in einem Gebiet auf unter 1:10000“ ist, milde ausgedrückt, fragwürdig, zumal der Anteil inapparent infizierter Personen dabei völlig unberücksichtigt bleibt. "WHO... eliminates leprosy by changing definitions and treatment duration, which has led to the belief that leprosy has indeed been eliminated and this jeopardizes adequate leprosy control and treatment" [54].

Das gilt auch für die "Elimination of a disease as a public health problem” [54]. Bei Lepra kommen eben nicht nur lange Latenzzeiten vor, sondern auch persistierende latente Infektionen, und die noch immer verbreitete Vorstellung, nur multibakterielle Fälle seien kontagiös, ist nicht aufrechtzuerhalten. Der größte Teil aller mit Lepra infizierten Menschen kann zwar u.U. Mykobakterien weitergeben, erkrankt aber selbst während des Lebens nie. Mitteleuropäer werden nur zu etwa 5\% krank. Dabei ist es nicht möglich, eine Grenze zwischen nicht endender Latenzzeit und dauernder Unempfindlichkeit zu bestimmen. In Anbetracht vieler inapparenter Infektionen ist die erwähnte „Definition“ der Elimination illusionär. Eine wissenschaftlich brauchbare Definition ist ganz anders [24]. Krankheit und Infektion werden getrennt: "Elimination of disease=reduction to zero of the incidence of a specified disease in a defined geographical area as a result of deliberate efforts, continued intervention measures are required" und "Elimination of infections = reduction to zero of the incidence of infection caused by a specific agent in a defined geographical area as a result of deliberate efforts; continued measures to prevent reestablishment of transmission are required".

„Eradikation“ ist nach den gleichen (Dahlem-) Kriterien definiert als "Permanent reduction to zero of the worldwide incidence of infection caused by a specific agent as a result of deliberate efforts. Intervention measures are not longer required." Daran gemessen, waren alle Ankündigungen einer Eradikation von Anfang an wissenschaftlich nicht seriös. Die von der WHO willkürlich angewendeten Benennungen täuschen [53-54].

Vor allem sind Grundvoraussetzungen der Eradikation nicht erfüllbar, denn der Mensch ist nicht einziger Wirt der Erreger, ansteckungsfähige Träger inapparenter Infektionen sind häufig. Regional unterschiedlich groß ist der Bevölkerungsanteil konstitutionell nicht erkrankender Individuen [27-29]. Lepra wird außerdem in aktuellen Endemiegebieten bei weitem zu wenig erkannt [53 - 56]. Die Zahl der Neumanifestationen nimmt vielerorts seit Jahren nicht, wie erwartet, ab [51 -54]. Lepra-Erreger sind durchaus außerhalb des Organismus, auch im Staub und im Boden, einige Zeit überlebensfähig [32]. Kaum einzuschätzen ist außerdem in manchen Gebieten die Häufigkeit weitgehend erscheinungsfreier kontagiöser Keimträger, bei denen mit späteren Rezidiven zu rechnen ist, nach insuffizienter Kurzzeit-MDT [49, 51].

Noch mehr: "Statements to the effect that MDT can interrupt transmission are unsubstantiated, as there are no convincing data which show this to be true. Evidence-based studies deny that effect of MDT on leprosy transmission" [54].

Das immer neue Auftreten gegen mehrere Mittel der MDT resistenter Bakterienstämme ist zwar durch neu entwickelte Medikamente aufzufangen und auch der Wechsel von billigen zu effektiveren MDT-Schemata könnte die Rückfallraten verringern. Aber die Vorstellung, die Lepra durch Chemotherapie auszurotten, ist aussichtslos [49]. Man müsste dazu nicht nur manifest Kranke, sondern alle infizierten Personen auffinden und behandeln. Der Aufwand wäre unermesslich: Z.B. werden bei Reihenuntersuchungen in Endemie-Gebieten, ohne dass immer dort auch manifest Kranke gefunden wurden, in hohen Prozentsätzen BakterienDNA in der für die Tröpfcheninfektion wesentlichen Nasenschleimhaut nachgewiesen. "The nose is considered to be a portal of entry, suggesting an aerial route for transmission through droplet infection. The evidence suggests that many individuals from endemic countries carry Mycobacterium leprae in their nasal cavities without having obvious symptoms of leprosy" [30, $31,57]$.

\section{Schlussfolgerung \\ $\nabla$}

Weltweite Eradikation der Lepra ist in unserer Zeit unmöglich, Elimination ist noch weitgehend Illusion [57]. Dies durch Bezeichnungen wie "Elimination as a public health problem" zu verschleiern, ist nicht ehrlich. Wirklich erreichbar ist nur die Kontrolle der Lepra [24] ("Reduction of disease incidence, prevalence, morbidity or mortality to a locally accepted level as a result of deliberate efforts”). Mehr ist das, was die WHO „Elimination“ nennt, nicht.

Besserung der therapeutischen Situation ist aus zwei ganz anderen Richtungen zu erwarten: 
Einerseits ist zwar die Fähigkeit des Organismus zur Auseinandersetzung mit Mykobakterien genetisch determiniert, aber nicht unveränderlich. Voraussichtlich werden sich in der global beweglichen Menschheit auch in anderen Regionen solche Genpolymorphismen im Bereich der angeborenen Immunität von selbst weiter durchsetzen, wie sie offenbar für den Rückgang der Lepra in Zentral-Europa am Ende des Mittelalters wesentlich waren [9].

Andererseits wird seit Langem an der Entwicklung spezifisch wirksamer Impfstoffe gearbeitet. Am Anfang standen Beobachtungen, die auf „Kreuzimmunität“ zwischen verschiedenen Mykobakterien-Infekten hinweisen. Befunde von Convit an einer früher lange Zeit weitgehend isolierten Einwanderergruppe in Venezuela ließen an eine Schutzwirkung der gegen Tuberkulose gerichteten BCG-Impfung gegen Lepra denken [36]. Noch ist zwar das Ziel, spezifische Immunisierung, nicht erreicht $[58,59]$. Aber wirksame Impfungen könnten einst angstfreie Koexistenz der Menschen mit Lepra-Erregern auch in bisherigen EndemieGebieten ermöglichen. Bis dahin müssen wir klar sehen, dass die Medizin, bildlich gesprochen, zwar schon viele Schlachten, aber noch lange nicht den Krieg gegen die Lepra gewonnen hat [60].

\section{Literatur}

1 Silva CA, Danelishvili L, McNamara M et al. Interaction of Mycobacterium leprae with human airway epithelial cells: adherence, entry, survival, and identification of potential adhesins by surface proteome analysis. Infect Immun 2013; 81: 2645-2659

2 Naves MdeM, Ribeiro FA, Patrocinio LG et al. Bacterial load in the nose and its correlation to the immune response in leprosy patients. Lepr Rev 2013; 84: 85-91

3 Hundeiker M, Perusquia-Ortiz AM, Bassukas ID. El futuro de la lepra: Ilusiones y realidad. Dermatol Rev Mex 2013; 57: 491 - 495

4 Wennberg-Hilger KK. Das seuchenhafte Auftreten von Lepra in einigen Küstenregionen West-Norwegens im 19. Jahrhundert. Mit einem ergänzenden Bericht über die entsprechende Situation in Schweden. Med. Inaug.-Diss Bonn: 2011

5 Agius-Ferrante A, Depasquale G, Bonnici $E$ et al. The leprosy eradication-project of Malta. Z Tropenmed Parasitol 1973; 24 (Suppl. 01): $49-52$

6 Briffa DV. 'Eradication' of leprosy from Malta. Lepr Rev 1987; 58: 87 89

7 Aranzazu N, Parra JJ, Cardenas M et al. Cojedes: a leprosy hyperendemic state. Int J Dermatol 2012; 51: 186-194

8 Alencar CH, Ramos AN Jr, Barbosa JC et al. Persisting leprosy transmission despite increased control measures in an endemic cluster in Brazil: the unfinished agenda. Lepr Rev 2012; 83: 344-353

9 Bassukas ID, Gaitanis G, Hundeiker M. Leprosy and the natural selection for psoriasis. Medical Hypotheses 2012; 78: 183-196

10 Singal A, Sonthalia S. Leprosy in post-elimination era in India: difficult journey ahead. Indian J Dermatol 2013; 58: 443 - 446

11 Han XY, Seo YH, Sizer KC et al. A new Mycobacterium species causing diffuse lepromatous leprosy. Am J Clin Pathol 2008; 130: 856-864

12 Gillis TP, Scollard DM, Lockwood DN. What is the evidence that the putative Mycobacterium lepromatosis species causes diffuse lepromatous leprosy? Lepr Rev 2011; 82: 205-209

13 Jessamine PG, Desjardins $M$, Gillis $T$ et al. Leprosy-like illness in a patient with Mycobacterium lepromatosis from Ontario, Canada. J Drugs Dermatol 2012; 11: 229-233

14 Cole ST, Eiglmeier K, Parkhill J et al. Massive gene decay in the leprosy bacillus. Nature 2001; 409: 1007-1011

15 Monot $M$, Honoré $N$, Garnier $T$ et al. On the origin of leprosy. Science 2005; 308: 1040 - 1042

16 Monot M, Honoré N, Garnier T et al. Comparative genomic and phylogeographic analysis of Mycobacterium leprae. Nat Genet 2009; 41: $1282-1289$

17 Achtman M. Insights from genomic comparisons of genetically monomorphic bacterial pathogens. Philos Trans R Soc Lond B Biol Sci 2012; 367: $860-867$

18 Meyers WM, Gormus BJ, Walsh GP. Nonhuman sources of leprosy. Int J Lepr Other Mycobact Dis 1992; 60: 477-480
19 Walsh GP, Meyers WM, Binford CH. Naturally acquired leprosy in the nine-banded armadillo: a decade of experience 1975-1985. J Leukoc Biol 1986; 40: 645-656

20 Truman R. Leprosy in wild armadillos. Lepr Rev 2005; 76: 198-208

21 Frota CC, Lima LN, Rocha AdaS et al. Mycobacterium leprae in six-banded (Euphractus sexcinctus) and nine-banded armadillos (Dasypus novemcinctus) in Northeast Brazil. Mem Inst Oswaldo Cruz 2012; 107 (Suppl. 01): 209-213

22 Clark BM, Murray CK, Horvath $L L$ et al. Case-control study of armadillo contact and Hansen's disease. Am J Trop Med Hyg 2008; 78: 962 - 967

23 Lane JE, Walsh DS, Meyers WM et al. Borderline tuberculoid leprosy in a woman from the state of Georgia with armadillo exposure. J Am Acad Dermatol 2006; 55: 714-716

24 Dowdle WR. The principles of disease elimination and eradication. Bull World Health Organ 1998; 76 (Suppl. 02): $22-25$

25 Mayr A. Eradikation und Tilgung von Seuchen. Dtsch Ärztebl 2006; 103 A: $3115-3118$

26 Hart BE, Tapping RI. Genetic Diversity of Toll-like Receptors and Immunity to M. leprae Infection. J Trop Med DOI 10.1155/2012/415057

27 Fava V, Orlova M, Cobat A et al. Genetics of leprosy reactions: an overview. Mem Inst Oswaldo Cruz 2012; 107 (Suppl. 01): 132 - 142

28 Cardoso CC, Pereira AC, de Sales MarquesC et al. Leprosy susceptibility: genetic variations regulate innate and adaptive immunity, and disease outcome. Future Microbiol 2011; 6: 533-549

29 Boldt AB, Sanchez MI, Stahlke ER et al. Susceptibility to leprosy is associated with M-ficolin polymorphisms. J Clin Immunol 2013; 33: 210 219

30 Klatser PR, van Beers S, Madjid B et al. Detection of Mycobacterium leprae nasal carriers in populations for which leprosy is endemic. J Clin Microbiol 1993; 31: 2947-2951

31 Naves MdeM, Ribeiro FA, Patrocinio LG et al. Bacterial load in the nose and its correlation to the immune response in leprosy patients. Lepr Rev 2013; 84: 85-91

32 Turankar RP, Lavania M, Singh $M$ et al. Dynamics of Mycobacterium leprae transmission in environmental context: Deciphering the role of environment as a potential reservoir. Infect Genet Evol 2012; 12 : $121-126$

33 Alter A, Grant A, Abel L et al. Leprosy as a genetic disease. Mamm Genome 2011; 22: 19-31

34 Fitness J, Tosh K, Hill AVS. Genetics of susceptibility to leprosy. Genes and Immunity 2002; 3: $441-453$

35 Johnson CM, Lyle EA, Omueti KO et al. Cutting edge: A common polymorphism impairs cell surface trafficking and functional responses of TLR1 but protects against leprosy. J Immunol 2007; 178: 7520-7524

36 Convit J, Gonzales CL, Rassi E. Estudios sobre lepra en el grupo étnico alemán de la Colonia Tovar, Venezuela. Internat J Leprosy 1952; 20 : $185-193$

37 Hohmann N, Voss-Böhme A. The epidemiological consequences of leprosy-tuberculosis co-infection. Math Biosci 2013; 241: 225 -237

38 De Sales Marques C, Brito-de-Souza VN, Guerreiro LT et al. Toll-like Receptor 1 N248S Single-Nucleotide Polymorphism Is associated with leprosy risk and regulates immune activation during mycobacterial infection. J Infect Dis 2013; 208: 120-129

39 Lydakis C, Ioannidou D, Koumpa I et al. Development of lepromatous leprosy following etanercept treatment for arthritis. Clin Rheumatol 2012; 31: 395-398

40 De Freitas MR, Said G. Leprous neuropathy. Handb Clin Neurol 2013; 115: $499-514$

41 Reinar LM, Forsetlund L, Bjrndal $A$ et al. Interventions for skin changes caused by nerve damage in leprosy. Sao Paulo Med J 2013; 131: 210

42 Rodriguez G, Pinto R, Gomez Y et al. Pure neuritic leprosy in patients from a high endemic region of Colombia. Lepr Rev 2013; 84: 41 - 50

43 Sales AM, Campos DP, Hacker MA et al. Progression of leprosy disability after discharge: is multidrug therapy enough? Trop Med Int Health 2013; 18: $1145-1153$

44 Scollard DM. The biology of nerve injury in leprosy. Lepr Rev 2008; 79: $242-253$

45 Scollard DM, Adams LB, Gillis TP et al. The Continuing Challenges of Leprosy. Clin Microbiol Rev 2006; 19: $338-381$

46 Bretan O, De Souza LB, Lastória JC. Laryngeal lesion in leprosy and the risk of aspiration. Lepr Rev 2007; 78: 80-81

47 Pallagatti S, Sheikh S, Kaur A et al. Oral cavity and leprosy. Indian Dermatol Online J 2012; 3: 101-104

48 Sharp D. Leprosy lessons from old bones. Lancet 2007; 369: 808-809 
49 Jacobson RR, Gatt P. Can leprosy be eradicated with chemotherapy? An evaluation of the Malta Leprosy Eradication Project. Lepr Rev 2008; 79: $410-415$

50 WHO Expert Committee on Leprosy. World Health Organ Tech Rep Ser. 2012. (968) World Health Organization; 1-61, 1 p following 61

51 Lockwood DN, Suneetha S. Leprosy: too complex a disease for a simple elimination paradigm. Bull World Health Organ 2005; 83: 230-235

52 Geluk A. Challenges in immunodiagnostic tests for leprosy. Expert Opin Med Diagn 2013; 7: 265-274

53 Naafs B. Treatment of Leprosy: science or politics? Tropical Medicine \& International Health 2006; 11: 268-278

54 Nsagha DS, Bamgboye EA, Assob JC et al. Elimination of leprosy as a public health problem by 2000 AD: an epidemiological perspective. Pan Afr Med J 2011; 9: 4 [Epub 2011 May 17]
55 Barreto JG, Guimarães LdeS, Frade MA et al. High rates of undiagnosed leprosy and subclinical infection amongst school children in the Amazon Region. Mem Inst Oswaldo Cruz 2012; 107 (Suppl. 01): 60-67

56 Beyene D, Aseffa A, Harboe $M$ et al. Nasal carriage of Mycobacterium leprae DNA in healthy individuals in Lega robi village, Ethopia. Epidemiol Infect 2003; 131: $841-848$

57 Silva KKMBK, Gunasekera CN, Fernando J. Clinical and histological activity after short duration multidrug therapy for leprosy. Sri Lanka J Dermatol 2003; 7: $22-23$

58 Duthie MS, Saunderson P, Reed SG. The potential for vaccination in leprosy elimination: new tools for targeted interventions. Mem Inst Oswaldo Cruz 2012; 107 (Suppl. 01): 190-196

59 Degang Y, Nakamura K, Akama T et al. Leprosy as a model of immunity. Future Microbiol 2014; 9: 43-54

60 Pattnaik S. Leprosy elimination: Battle won, but the war remains. Natl Med J India 2013; 26: $57-58$ 